\title{
The Marshallian Consumer
}

\author{
By TAPAN BISWAS \\ University of Manchester
}

Ever since the publication of Principles (Marshall, 1890), the Marshallian assumption that the marginal utility of money is constant has been the source of many debates and confusions. Consequently, the Marshallian demand function has been interpreted in different ways by different authors. Some of the exponents of the Marshallian demand theory (e.g. Samuelson, 1942; Green, 1971) assume a Paretian framework, in which the consumer shops for the day with a predetermined expenditure level. They derive the form of the utility function that is consistent with the assumption that the marginal utility of money (expenditure) is invariant with respect to the prices the consumer has to pay. This is essentially a Paretian exercise, the origin of which may be traced back to Pareto (1892). Although the exercise is interesting, it is hard to believe that Marshall built his demand theory on the weak foundation of a highly restricted class of utility functions. There are, of course, well-known alternative interpretations (e.g. Friedman, 1949; Bailey, 1954). In his discussion on the nature of the demand function (and the theory of consumer's surplus), Marshall assumed "other things" to be equal. In other words, the "conjuncture" or the circumstances are to be considered as invariant with respect to the change in price. Friedman (1949) interpreted this as implying a movement along the indifference surface. However, this interpretation is not very satisfactory. Friedman himself admitted that the case of Giffen good does not fit in his interpretation of Marshall. Moreover, the marginal utility of money does not, in general, remain constant when the consumer shifts his position on the indifference curve accompanied by a compensated variation in income.

In this paper we provide an alternative interpretation of the Marshallian assumption that the marginal utility of money is constant. We support our exegesis by proper textual documentation. It seems that most of the confusions arose in trying to interpret Marshall in the Paretian framework of a budget-constraint model. The literature talks about Walras-Marshall-Pareto demand theory (see Wilson, 1939) without realizing that, even in the last edition of his Principles, Marshall never cited any work of Walras or Pareto in the context of the demand theory although he referred to their works in other contexts.

A few years after the first edition of Principles was published, Sanger (1895) wrote a survey article on the recent contributions to mathematical economics. Towards the end of the article he was explaining Barone's (1894) interpretation of the difference between the Walrasian consumer's surplus and the Marshallian consumer's surplus. Barone proved that they 
change by an equal amount if the variation in prices is small. Quoting directly from Sanger (1895, p. 125):

Professor Marshall assumes that $m$ remains constant .... According to Professor Walras, the individual spends an amount of money $M$, while Professor Marshall makes him spend $M+\delta M$ where $\delta M$ is evidently given by $\delta M=$ $r_{a} \Delta p_{a}+p_{a} \delta r_{a}+r_{b} \Delta p_{b}+p_{b} \delta r_{b}+r_{c} \Delta p_{c}+p_{c} \delta r_{c}$

Here $\delta M$ is the change in money expenditure when the prices change, but to the consumer the marginal utility of money remains the same. Evidently Marshall agreed to this interpretation, because he referred to this section of Sanger's article in his Principles (p. 109n) (all page references are to the eighth edition, reset in 1949). Therefore, the expenditure level of a Marshallian consumer may be affected by price changes, but the marginal utility of money remains unaffected. Marshall recognized that, owing to joy and excitement, the rate of expenditure incurred by a consumer over a certain period may well exceed the rate that his ability permits. Afterwards, the consumer should become aware of this possibility and should learn to control his expenditure. Marshall's example of a young couple (p. 99) may be cited in this context. Thus, although budgetary considerations gain prominence in the long run, the behaviour of a consumer during a certain period need not be constrained by a fixed budget.

The behaviour pattern of a Marshallian consumer that emerges from the above discussion is different from that of a Paretian consumer, whose behaviour was elaborately studied by Hicks (1939). A Marshallian consumer starts shopping for the day with a predetermined rate of exchange between money and utility. If the consumer is willing to exchange one unit of money for $\lambda$ units of utility then, obviously, $\lambda$ is the marginal utility of money. In equilibrium, the marginal utility of money must be equal to the marginal utility of expenditure.

We may present the consumer's decision problem as:

$$
\text { Maximize } z=u(x)-\lambda p^{\prime} x
$$

where $x$ and $p$ are the consumption and the price vectors respectively, and $\lambda$ is the marginal utility of money. The values of $\lambda$ and $p$ are known. Equation (1) follows from Marshall's doctrine of maximum net satisfaction (p. 276, also Notes XII and XIV in the Mathematical Appendix). According to Marshall, this doctrine holds good only at the micro-level. It does not apply to the society as a whole. In the context of (1), one may also cite an interesting sentence from Principles. While discussing the decision problem of a young couple, Marshall wrote: "They strive to adjust their parings down so that the aggregate loss of utility may be a minimum, and the aggregate of utility that remains to them may be a maximum". (p. 99). If $x^{*}$ is the solution for equation (1), then it can be shown that $\lambda p x^{*}$ is minimum subject to the attainment of $z^{*}=u\left(x^{*}\right)$.

Given the decision problem of a Marshallian consumer, the first-order condition for a maximum is

$$
u_{i}=\lambda p_{i} \text {. }
$$

Since $\lambda$ is predetermined by the second-order condition, $U$ (the Hessian of the utility function) should be negative definite. If we want to guarantee that 
the second-order condition is satisfied by all $x$ satisfying equation (2), for all possible values of $\lambda$ and $p$, the most natural assumption to be made is: $U$ is negative definite everywhere. This implies that the utility function is strictly concave everywhere. We shall assume this to be true for a Marshallian consumer. If the utility function is strictly separable and the law of diminishing marginal utility holds $\left(u_{i i}<0\right)$, then $U$ is trivially negative definite everywhere. It is widely held that Marshall assumed a separable utility function. It is true that, throughout the Mathematical Appendix, he was assuming a separable utility function. But in the text Marshall admitted: "....we cannot say that the total utility of two together is equal to the sum of the total utilities of each separately" (p. 109). In this paper we hope to prove that the separability assumption is not essential in the context of the Marshallian demand theory.

For the Marshallian consumer, we have assumed that $U$ is negative definite. One should compare this with the assumption of quasi-concavity in the context of the Hicksian demand theory where the consumer's expenditure level $(E)$ is predetermined. The second-order condition (implying local quasi-concavity at the point of equilibrium) for the Hicksian consumer is weaker: $d x^{\prime} U d x<0$ provided $p^{\prime} d x=0$. Therefore, if the utility function of a consumer is merely quasi-concave, we may encounter the possibility that a situation $(x, p, E, \lambda)$ is an optimal solution with respect to the Hicksian behaviour, but not so if the consumer behaves in the Marshallian way. This will happen whenever $d x^{\prime} U d x$ is positive for some $d x$, although $d x^{\prime} U d x$ is strictly negative for all $d x$ satisfying $p^{\prime} d x=0$. This is the case if the utility function, at the point of equilibrium for a Hicksian consumer, is not even weakly concave although it is a quasi-concave function. If $U$ is negative definite everywhere (which implies strict concavity of $u$ ), any Hicksian equilibrium is also an equilibrium for a Marshallian consumer. Suppose $(x, p, E, \lambda)$ is an equilibrium situation for a Hicksian consumer. If he wants to behave like a Marshallian consumer treating the same $\lambda$ as the predetermined rate of exchange between money and utility, the equilibrium situation remains unaltered. The first-order conditions are the same. Marshallian second-order conditions are satisfied because $U$ is negative definite everywhere.

Since $U$ is negative definite, we are allowed to write (2) in the following form:

$$
x_{1}=\theta_{i}\left(p_{1}, p_{2}, \ldots, p_{n} ; \lambda\right) .
$$

the mapping defined by (3) is globally unique. The actual expenditure incurred by the consumer is

$$
E=\sum_{i=1}^{n} p_{i} \theta_{i}\left(p_{1}, p_{2}, \ldots, p_{n} ; \lambda\right)
$$

The prices being given, the actual expenditure level depends upon the value of $\lambda$, the minimum amount of utility that must be obtained in exchange of one unit of money. This is different from the Walras-Pareto-Hicks theory of consumer's behaviour where the magnitude of $\lambda$ depends upon the prices and the predetermined expenditure level. 
Although a Marshallian consumer is not subject to any budget constraint in the short run, such a restriction must prevail in the long run. We are using the terms short run and long run in the conventional rather than in the Marshallian sense. Consider a decision period (e.g. a Hicksian week) for the consumer. If the consumer makes his purchases on the basis of a predetermined rate of exchange between money and utility, at the end of the week he will find that he has spent a certain sum $(E)$ over the week. On the other hand, he is expected to form an idea as to how much he should have spent $\left(E^{*}\right)$, after he has observed his earnings and the events during the week that affect his future. If the actual expenditure $(E)$ is different from what he should have spent $\left(E^{*}\right)$ he is expected to revise the rate of exchange between money and utility which, to him, is equal to the marginal utility of money. The consumer's actual expenditure will be different in the next period.

It is extremely important to examine the stability of this process. If the prices and the desired level of expenditure remain the same (the financial position of the consumer being stable), will this process of revision ultimately equalize $\lambda$ and actual expenditure $(E)$ to their desired level $\left(\lambda^{*}, E^{*}\right)$ ? In terms of Hicksian analysis, $\lambda^{*}$ is the marginal utility of money when the predetermined expenditure level is $E^{*}$.

Let us consider the following adjustment process for $\lambda$ :

$$
d \lambda / d t=f\left(E-E^{*}\right)
$$

where $f$ is a sign-preserving continuous function and $E^{*}$ is the desired level of expenditure which is assumed to be constant over time. In general, $E^{*}$ depends on many factors, including the consumer's income and wealth, market prices and $\lambda$. We are introducing an element of artificiality by treating $E^{*}$ as a constant. In fact, we are carrying out an experiment in an artificial environment, like most of the experiments in the physical sciences, to show that, if the desired expenditure level remains constant over time, then actual expenditure will gradually converge to the desired level. In other words, we shall show that the consumer's decision process, as outlined above, is inherently stable. For any exogenous price vector, we may obtain the following from (2)-(4):

$$
\frac{\delta E}{\delta \lambda}=\sum_{i=1}^{\dot{\sum}} p_{i} \frac{\delta \omega_{1}}{\delta \lambda}=p^{\prime} U^{-1} p<0 .
$$

We have used the relation $\delta x / \delta \lambda=U^{-1} p$ which follows from (2) for a given price vector. Since the consumption vector $x$ is chosen in such a way that the net utility $z$ is maximum, $U$ is negative definite by the second-order condition.

Consider Figure 1. At an initial price vector $p^{0}$ and a desired expenditure level $E^{*}$, the consumer is in equilibrium with $\lambda=\lambda^{*}\left(p^{0}\right)$. For $p=p^{0}$, the $E^{0} E^{0}$ curve relates the actual expenditure levels to different values of $\lambda$. If $\lambda=\lambda^{*}\left(p^{0}\right)$ with $p=p^{1}, E$ is greater than $E^{*}$. Through the process of revising $\lambda$ according to equation (5), $\lambda^{*}\left(p^{1}\right)$ will be attained in the long run. Note that, in order to guarantee that the second-order condition for the Marshallian maximization problem is always satisfied, $U$ is assumed to be negative 


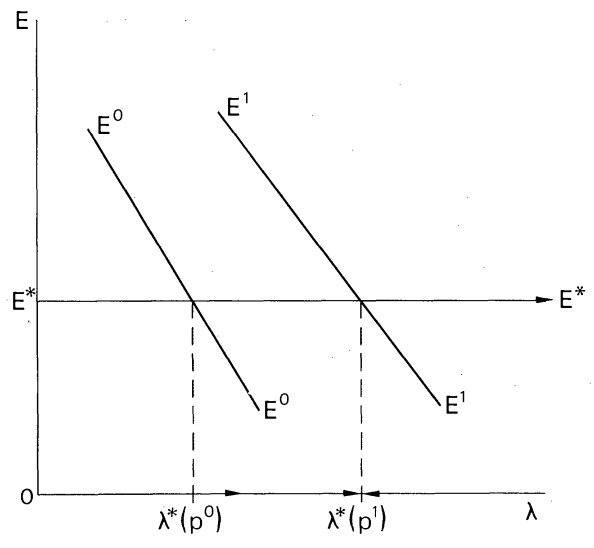

FIGURE 1

definite everywhere. Therefore, if for $\left(E^{*}, p^{1}\right)$ an optimal interior solution exists in the Hicksian decision problem, $E^{1} E^{1}$ must intersect $E^{*} E^{*}$.

Marshall claimed that an increase in price will reduce the demand for a commodity if the marginal utility of money is constant. This follows immediately if the utility function is separable. Is this also true when the utility function is not separable? The answer is "yes". If $\lambda$ is treated as a constant, equation (2) yields

$$
\frac{\delta x_{i}}{\delta p_{i}}=\frac{\lambda\left|U_{i i}\right|}{|U|}<0
$$

where $\left|U_{i i}\right|$ is the principle minor associated with $u_{i i}$. In the Marshallian decision process, the second-order condition for a maximum requires $U$ to be negative definite. This explains equation (7).

\section{II}

The Marshallian assumption that the marginal utility of money is constant is an assumption regarding the initial impact of the changed circumstances upon $\lambda$. In the previous section this has been interpreted as a short-run assumption. If the amount of actual expenditure is different from what is desired, a revision in $\lambda$ takes place. When $E^{*}$ remains constant over time, the magnitude of $\lambda$ is ultimately adjusted to make $E$ coincide with $E^{*}$. A change in prices leave $\lambda$ unaltered only for a short period. Ultimately, $\lambda$ must change with a change in $p_{i}$. We have just shown that, for a Marshallian consumer, the demand curve must have a negative slope in the short run. Is this also true if the consumer, with his desired expenditure level unaltered, immediately takes the change in $\lambda$ into account? The answer is "no". In the situation where $\lambda$ is adjusted instantaneously, the decision problem of the consumer is still presented by equation (1). Only the predetermined value of $\lambda$ is always such as to equalize $E$ with $E^{*}$. The Marshallian demand curve degenerates into a Hicksian demand curve (a) in the long run if $E^{*}$ is 
constant, and (b) in the case of an instantaneous adjustment in $\lambda$. In the second case, the graph of the function $f$ in (5) coincides with the axis representing $d \lambda / d t$. It is well understood, following the example of Sir Robert Giffen (1879), that when the change in $\lambda$ is taken into account a section of the demand curve may have a positive slope. This does not contradict the Marshallian proposition that, when $\lambda$ is predetermined, the slope of the demand curve must be negative.

Marshall's explanation of the Giffen phenomenon is different from the usual one:

There are, however, some exceptions. For instance, as Sir R. Giffen has pointed out, a rise in the price of bread makes so large a drain on the resources of the poorer labouring families and raises so much the marginal utility of money to them, that they are forced to curtail their consumption of meat and the more expensive farinaceous foods: and, bread being still the cheapest food which they can get and will take, they consume more and not less of it. [pp. 109-110]

In other words, according to Marshall, $x_{i}$ may behave like a Giffen good if both $\delta x_{i} / \delta \lambda$ and $\delta \lambda / \delta p_{i}$ are positive, and $\delta \lambda / \delta p_{i}$ is a large number. Moreover, $\delta \lambda / \delta p_{i}$ will assume a large value if $\delta E / \delta p_{i}$ evaluated at $\lambda=\lambda^{\circ}$ is large. Remember, in this section we are assuming the desired expenditure level of the Marshallian consumer to be fixed, and any change in, $\lambda$, owing to a variation in $p_{i}$, is fully taken into account by the consumer. The term

$$
\delta E /\left.\delta p_{i}\right|_{\lambda=\lambda^{\circ}}
$$

measures the change in the expenditure level the consumer will have to bear, if he does not revise $\lambda$ following the change in $p_{i}$.

Marshall's explanation will be better understood in terms of the cardinalistic decomposition of the price effect. Hicks (1956, pp. 13-14) explained the cardinalistic decomposition in the following way. Consider equation (2) and a change in $p_{i}$. The demand for $x_{i}$ will change because (a) $p_{i}$ has changed to $p_{i}+\delta p_{i}$ and (b) $\lambda$ has changed. The change in the demand for $x_{i}$ owing to the first reason is called the direct effect. The change in the demand for $x_{i}$ owing to the change in $\lambda$ is called the indirect effect. It has been shown in the Appendix that from equation (2) one can obtain the following expression:

$$
\frac{\delta x_{i}}{\delta p_{i}}=\frac{\lambda\left|U_{i i}\right|}{|U|}+\left.\left.\frac{\delta x_{i}}{\delta \lambda}\right|_{p=p_{0}} \frac{\delta \lambda}{\delta p_{i}}\right|_{E=E^{*}} .
$$

The first term in the right-hand side is the direct effect and the next term is the indirect effect. The direct effect is nothing but the slope of the Marshallian demand curve. The slope is negative by equation (7). A change in $p_{i}$ shifts the Marshallian demand curve because of a variation in $\lambda$. Equation (8) has nothing to do with the slope of the new (short-run) Marshallian demand curve. It merely explains the slope of the curve derived from the long-run relationship between $p_{i}$ and $x_{i}$ if the level of desired expenditure remains the same.

Equation (8) makes it clear that a Giffen situation may arise if and only if 
the indirect effect is positive and is larger than the direct effect in the absolute magnitude. In the Appendix, we have proved the following:

$$
\begin{aligned}
\left.\frac{\delta \lambda}{\delta p_{i}}\right|_{E=E^{*}} & =-\left.\left(x_{i}+\left.\sum_{j=1}^{n} p_{j} \frac{\delta x_{j}}{\delta p_{i}}\right|_{\lambda=\lambda^{\circ}}\right) \frac{\delta \lambda}{\delta E^{*}}\right|_{p=p^{0}} \\
& =-\left.\left.\frac{\delta E}{\delta p_{i}}\right|_{\lambda=\lambda^{\circ}} \frac{\delta \lambda}{\delta E^{*}}\right|_{p=p^{\circ}} .
\end{aligned}
$$

Since $U$ is negative definite, $\delta \lambda / \delta E^{*}=-|U| /|S|<0$, where $|S|$ is the well-known bordered Hessian in Hicks (1939). When $U$ is negative definite, the signs of both $|U|$ and $|S|$ are determined by $(-1)^{n}$ in equilibrium with the restriction $E=E^{*}$ satisfied. If the consumer immediately adjusts the magnitude of $\lambda$ in response to a variation in the desired expenditure level, $E^{*}$ and $\lambda$ vary in opposite directions. The larger is the sum the consumer can afford to spend, the smaller is the marginal utility of money to him. In the words of Marshall, "the richer a man becomes the less is the marginal utility of money to him" (p. 81).

The term $\delta \lambda / \delta E^{*}$ being negative, it is obvious from (9) that the sign and the magnitude of $\delta \lambda / \delta p_{i}$ depends on

$$
\delta E /\left.\delta p_{i}\right|_{\lambda=\lambda^{0}} \text {. }
$$

This was correctly observed by Marshall. It is also apparent from (8) that the indirect effect will be positive and relatively large if both $\delta x_{i} / \delta \lambda$ and $\delta \lambda / \delta p_{i}$ are positive and the latter is significantly large. Marshall was absolutely correct in his intuitive understanding of the Giffen phenomenon.

Hicks (1956, pp. 12-15) argued that the division of the price effect into direct and indirect effects is conceptually related to the division between substitution and income effects. This may be shown very easily. Equations (8) and (9) yield

$$
\frac{\delta x_{i}}{\delta p_{i}}=\frac{\lambda\left|U_{i i}\right|}{|U|}-\left.\left.\frac{\delta E}{\delta p_{i}}\right|_{\lambda=\lambda^{\circ}} \frac{\delta x_{i}}{\delta E^{*}}\right|_{p=p^{0}} .
$$

compare this with the Slutsky equation:

$$
\frac{\delta x_{i}}{\delta p_{i}}=\frac{\lambda\left|S_{i i}\right|}{|S|}-\left.x_{i} \frac{\delta x_{i}}{\delta E^{*}}\right|_{p=p^{0}}=x_{i i}-\left.\left.\frac{\delta E}{\delta p_{i}}\right|_{u=u^{0}} \frac{\delta x_{i}}{\delta E^{*}}\right|_{p=p^{0}} .
$$

Note that we have used the relation

$$
x_{i}=\delta E /\left.\delta p_{i}\right|_{u=u^{0}}
$$

which is well known. The conceptual affinity between the direct and the substitution effects is obvious. The indirect effect is the product between the derivative of $x_{i}$ with respect to $E^{*}$ (Hicksian income derivative) and the increment in expenditure required to keep $\lambda$ constant. The income effect has the same interpretation, except that the increment in expenditure is such that the same level of satisfaction is maintained. Therefore the Marshallian explanation of Giffen phenomenon in terms of the direct and the indirect effects is conceptually very similar to the Hicksian explanation in terms of the substitution and the income effects. 
We are at the end of our discussion. Before we conclude, it seems appropriate to pay some attention to Marshall's comment that expenditure on any particular commodity forms only a small part of the consumer's total expenditure (pp. 278-280; 693). According to Marshall, this explains why $\lambda$ may be treated as a constant following a change in $p_{i}$. It seems, when Marshall made this comment, he was thinking in terms of a separable utility function. If the utility function is separable and $\lambda$ is held constant, a change in $p_{i}$ will only affect the expenditure on the $i$ th commodity. The expenditure on other items remains unchanged. The expenditure on the $i$ th commodity being only a small fraction of the total expenditure (both before and after the price change), the percentage change in the total expenditure is small. In this case a consumer, according to Marshall, will not make any instantaneous adjustment in $\lambda$, but will wait to see the future course of the prices and his earnings. So a change in $p_{i}$ will not have an instant impact on $\lambda$. This behavioural assumption is, of course, based on the presumption that the consumer has command over a large sum of money and can afford to wait and see (pp. 278-280). According to our interpretation, Marshall's comment does not imply that

$$
\delta \lambda /\left.\delta p_{i}\right|_{E=E^{*}}
$$

is negligible if $p_{i} x_{i} / E^{*}$ is small. It merely refers to a situation where it is reasonable to assume that a change in $p_{i}$ leaves $\lambda$, the rate of exchange between money and utility, unaltered during the period in question.

There are quite a few things in the Marshallian demand theory that are far from being satisfactorily explained to this date. But prior to any discussion on them, we must agree upon the nature and the motive of a Marshallian consumer. We claim to have him described satisfactorily. That is all we wanted.

\section{ACKNOWLEDGEMENTS}

I am indebted to my colleagues at the University of Manchester, particularly to I. Steedman, M. Parkin and Pat Minford. I am also grateful to the referee of this journal for some useful suggestions. It is needless to say that the responsibility for any error lies entirely with me.

\section{APPENDIX}

In this appendix, we shall derive the relationships expressed in equations (8) and (9). From (2) in the text we obtain, for a given $p, U \delta x=p \delta \lambda$. Applying Creamers's rule we obtain

$$
\left.\frac{\delta x_{i}}{\delta \lambda}\right|_{p=p^{0}}=\frac{1}{|U|} \quad\left|\begin{array}{ccccc}
u_{11} & \ldots & p_{1} & \ldots & i_{1 n} \\
\cdot & & \cdot & & \cdot \\
\cdot & & \cdot & & \cdot \\
\cdot & & \cdot & & \cdot \\
u_{n 1} & \ldots & p_{n} & \ldots & u_{n n}
\end{array}\right|
$$


In a situation where $\lambda$ is instantaneously adjusted to make $E=E^{*}, U \delta x=p \delta \lambda+\lambda \delta p$. Therefore, for a change in $p_{i}$ only,

(A2) $\left.U \frac{\delta x}{\delta p_{i}}\right|_{E=E^{*}}=\left.p \frac{\delta \lambda}{\delta p_{i}}\right|_{E=E^{*}}+\lambda e_{i}$,

where $e_{i}$ is a vector with $i$ th element unity and all other elements zero. From (A1) and (A2) we obtain

$$
\left.\frac{\delta x_{i}}{\delta p_{i}}\right|_{E=E^{*}}=\left.\left.\frac{\delta x_{i}}{\delta \lambda}\right|_{p=p^{0}} \frac{\delta \lambda}{\delta p_{i}}\right|_{E=E^{*}}+\lambda \frac{\left|U_{i i}\right|}{|U|}
$$

This is equation (8) in the text. Let us now proceed to derive equation (9). In case of instantaneous adjustment in $\lambda$, the constraint $p x=E^{*}$ must be satisfied. This together with (2) yields

$$
\left[\begin{array}{cccc}
u_{11} & \ldots & u_{1 n} & p_{1} \\
\cdot & & \cdot & \cdot \\
\cdot & & \cdot & \cdot \\
\cdot & & \cdot & \cdot \\
u_{n 1} & \ldots & u_{n n} & p_{n} \\
p_{1} & \ldots & p_{n} & 0
\end{array}\right] \quad\left[\begin{array}{c}
\frac{\delta x}{\delta p_{i}} \\
-\frac{\delta \lambda}{\delta p_{i}}
\end{array}\right]=\left[\begin{array}{c}
\lambda e_{i} \\
-x_{i}
\end{array}\right]
$$

therefore

$$
\begin{aligned}
& \text { (A3) }\left.\frac{\delta \lambda}{\delta p_{i}}\right|_{E=E^{*}}=x_{i} \frac{\left|S_{n n}\right|}{|S|}-\frac{\lambda}{|S|}\left|\begin{array}{ccc}
u_{11} & \ldots & i_{1 n} \\
\cdot & & \cdot \\
\cdot & & \cdot \\
\cdot & & \cdot \\
u_{i-1,1} & \ldots & u_{i-1, n} \\
u_{i+1,1} & \ldots & u_{i+1, n} \\
\cdot & & \cdot \\
\cdot & & \cdot \\
\cdot & & \cdot \\
u_{n 1} & \ldots & u_{n n} \\
p_{1} & \ldots & p_{n}
\end{array}\right|(-1)^{n+i+1} \\
& =x_{i} \frac{\left|S_{n n}\right|}{|S|}-\frac{\lambda}{|S|}\left|\begin{array}{ccc}
u_{11} & \ldots & u_{1 n} \\
\cdot & & \cdot \\
\cdot & & \cdot \\
\cdot & & \cdot \\
u_{i-1,1} & \ldots & u_{i-1, n} \\
p_{1} & \ldots & p_{n} \\
u_{i+1,1} & \ldots & u_{i+1, n} \\
\cdot & & \cdot \\
\cdot & & \cdot \\
\cdot & & \cdot \\
u_{n 1} & \ldots & u_{n n}
\end{array}\right|(-1)^{2 n+1} \quad \begin{array}{l}
\text { (using elementary } \\
\text { matrix) }
\end{array}
\end{aligned}
$$

Originally published in Economica (1977) 44, 47-56 


$$
\begin{aligned}
& =x_{i} \frac{\left|S_{n n}\right|}{|S|}+\frac{\lambda}{|S|} \sum_{j} p_{j}\left|U_{i j}\right|(-1)^{i+j} \\
& =\left(x_{i}+\sum_{j} p_{i} \frac{\lambda\left|U_{i j}\right|}{|U|}(-1)^{i+j}\right) \frac{\left|S_{n n}\right|}{|S|} \quad\left(\text { since }\left|S_{n n}\right|=|U|\right)
\end{aligned}
$$

Next, note that

$$
\left.\frac{\delta \lambda}{\delta E^{*}}\right|_{p=p^{0}}=-\frac{\left|S_{n n}\right|}{|S|} .
$$

Further,

$$
\left.\frac{\delta x_{j}}{\delta p_{i}}\right|_{\lambda=\lambda^{0}}=\lambda \frac{\left|U_{i j}\right|}{|U|}(-1)^{i+j}
$$

by symmetry $\left.\left|U_{i i}\right|=\left|U_{i j}\right|\right)$. Therefore, by (A3),

$$
\left.\frac{\delta \lambda}{\delta p_{i}}\right|_{E=E^{*}}=-\left.\left(x_{i}+\left.\sum_{j} p_{i} \frac{\delta x_{j}}{\delta p_{i}}\right|_{\lambda=\lambda^{0}}\right) \frac{\delta \lambda}{\delta E^{*}}\right|_{p=p^{0}} .
$$

This is equation (9) in the text. The expression within the brackets is the compensating variation required to keep $\lambda$ unchanged.

\section{REFERENCES}

BAILEY, M. (1954). The Marshallian demand curve. Journal of Political Economy, 62, 255-266.

BARONE, E. (1894). Sulla consumer's rent. Giornale degli Economisti, 9, 407-435.

EDgeworth, F. T. (1894). Professor J. S. Nicholson on consumer's rent. Economic Journal, 4, $151-158$.

Friedman, M. (1949). The Marshallian demand curve. Journal of Political Economy, 57, 463-495.

GifFEN, R. (1879). Economic Inquiries and Studies: London: George Bell and Sons.

Green, H. A. J. (1971). Consumer Theory. Baltimore: Penguin.

Hicks, J. R. (1939). Value and Capital. London: Oxford University Press.

- (1956). A Revision of Demand Theory. London: Oxford University Press.

Marshall, A.(1890). Principles of Economics. (8th ed., 1920; reset 1949; Papermac ed., 1962.) London: Macmillan.

Nicholson, J. S. (1894). The measurement of utility by money. Economic Journal 4, 342-347. PARETo, V. (1892). Considerazione sui princippii fondamentali - dell'economia politica pura. Giornale degli Economisti, 4, 486-512.

SAmuelson, P. A. (1942). Constancy of the marginal utility of income. In Studies in Mathematical Economics and Econometrics (O. Lang et al., eds), pp. 95-98. Chicago: University Press.

SANGER, C. P. (1895). Recent contributions in mathematical economics. Economic Journal, 5, 113-128.

Wilson, E. B. (1939). Pareto versus Marshall. Quarterly Journal of Economics, 43, 645-649. 